\title{
Aeroheating Analysis for the Mars Reconnaissance Orbiter with Comparison to Flight Data
}

\author{
Derek S. Liechty ${ }^{*}$ \\ NASA Langley Research Center, Hampton, VA 23681
}

\begin{abstract}
The aeroheating environment of the Mars Reconnaissance Orbiter (MRO) has been analyzed using the Direct Simulation Monte Carlo and free-molecular techniques. The results of these analyses were used to develop an aeroheating database to be used for the pre-flight planning and the in-flight operations support for the aerobraking phase of the MRO mission. The aeroheating predictions calculated for the MRO include the heat transfer coefficient $\left(C_{H}\right)$ over a range of angles-of-attack, side-slip angles, and number densities. The effects of flow chemistry were also investigated. Flight heat flux data deduced from surface temperature sensors have been compared to pre-flight predictions and agree favorably.
\end{abstract}

\section{Nomenclature}

$C_{H} \quad=$ heat transfer coefficient, $C_{H}=q /\left(0.5 \rho_{\infty} V_{\infty}{ }^{3}\right)$

$c_{p} \quad=$ specific heat

$k \quad=$ thermal conductivity

$n_{\infty} \quad=$ free stream number density

$q \quad=$ surface heat transfer rate

$T_{\infty} \quad=$ free stream temperature

$V_{\infty} \quad=$ free stream velocity magnitude

$\alpha \quad=$ angle-of-attack

$\beta \quad=$ side-slip angle

$\varepsilon \quad=$ emissivity

$\lambda_{\infty} \quad=$ free stream mean free path

$\rho_{\infty} \quad=$ free stream density

$\rho_{\mathrm{s}} \quad=$ solar cell substrate density

\section{Introduction}

T

he Mars Reconnaissance Orbiter (MRO) was launched on August 12, 2005. It has delivered to Mars orbit a payload to conduct remote sensing science observations, identify and characterize sites for future landers, and provide critical telecom/navigation relay capability for follow-on missions. Figure 1 illustrates the MRO passing through the Martian atmosphere during the aerobraking phase of its mission. During the one Martian year (687 Earth days) primary science phase, the orbiter will acquire visual and near-infrared highresolution images of the planet's surface, monitor atmospheric weather and climate, and search the upper crust for evidence of water. After the science phase is completed, the orbiter will provide telecommunications support for spacecraft launched to Mars in the 2007 and 2009 opportunities. The primary mission ends on December 31, 2010, approximately 5.5 years after launch.

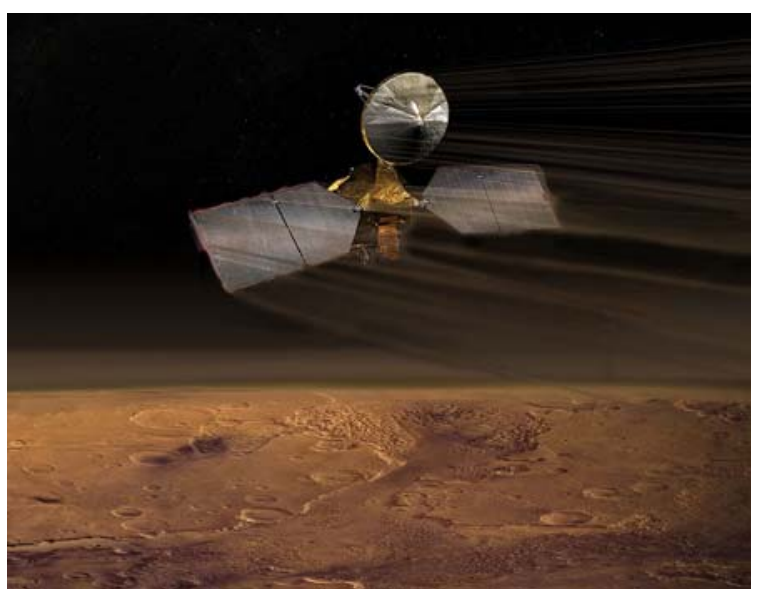

Figure 1. MRO in aerobraking phase.

*Aerospace Engineer, Aerothermodynamics Branch, m/s 408A.

1 of 8 
After Mars insertion, the aerobraking phase started with a series of walk-in maneuvers. The strategy was to gradually bring the periapsis altitude down to a level such that the dynamic pressure and heating rate satisfy pre-set corridor conditions for aerobraking main phase. While the periapsis altitude is within the upper bound of the Martain atmosphere, the spacecraft will be experiencing deceleration induced by atmospheric drag. In about six months, the orbit period will be reduced to about 2 hours from a 35 -hour initial orbit period. The total energy saving is equivalent to about $1200 \mathrm{~m} / \mathrm{s}$ in $\Delta U$.

All of the aerobraking is taking place at altitudes where the densities are sufficiently low that the flow is in the rarefied transitional regime. To accurately predict the aeroheating characteristics of the spacecraft in the rarefied transitional regime, the Direct Simulation Monte Carlo (DSMC) and free molecular techniques were used. The results from the calculations were used to create the aeroheating database of the spacecraft. Heating rates have been computed from thermocouple data collected in flight. These results are also presented and discussed.

Flight operations support is also being given throughout the aerobraking maneuver. Temperature sensor data, along with atmospheric data, is taken from the MRO and heating rates at specific sensor locations are calculated. These procedures and early samples are also presented herein.

\section{Computational Methods}

The DSMC calculations were performed using DDAC, the parallel implementation of the program DAC (DSMC Analysis Code). ${ }^{1,2}$ In DAC, the gas molecular collisions are modeled using the variable-hard-sphere (VHS) model developed by $\mathrm{Bird}^{3}$, and the Larsen-Borgnakke model is used for internal energy exchanges. The geometry surface is represented by unstructured triangular elements that are embedded in a two-level Cartesian grid for the flow field calculation. The solution from the first level of grid cells, which are uniform in size, is used for grid refinement to create the second-level cells. The grid is refined based on local conditions, thus allowing the program to meet the spatial resolution requirements without excessive global refinement. The grid cells are typically refined such that on average the second-level cells have dimensions less than the local mean free path. The local simulation parameters are set such that there are nominally 10 simulated molecules in each cell, and the local time step is typically dictated by the local flow time for the problems considered. The number of time steps until steady state was set to 5000, followed by enough sampling time steps to allow most of the surface triangular elements to accumulate at least 1000 molecular collisions (this was the lower limit for small surface elements; most surface elements had on the order of a few million molecular impacts), resulting in a total of somewhere on the order of 30,000 steady state time steps.

For all calculations, the wall collisions were assumed to be fully diffusive, i.e., an accommodation coefficient of one was specified, with the spacecraft wall temperature at a constant $300 \mathrm{~K}$. The composition of Mars atmosphere was assumed to be $95.37 \% \mathrm{CO}_{2}$ and $4.63 \% \mathrm{~N}_{2}$ by mole with a free stream temperature of $144.77 \mathrm{~K}$ and velocity of $4811.0 \mathrm{~m} / \mathrm{s}$ for densities below $100 \mathrm{~kg} / \mathrm{km}^{3}$, decreasing to $3611 \mathrm{~m} / \mathrm{s}$ at a density of approximately $350 \mathrm{~kg} / \mathrm{km}^{3}$. The computational geometry shown in Fig. 2 was derived from a CAD file provided by Lockheed Martin Astronautics (LMA) and represents the best pre-flight estimate of the nominal aerobraking configuration. Special care was taken to accurately represent the science modules for aeroheating analysis, as show in Fig. 3. Free stream conditions for all number densities simulated can be viewed in Table 1. At each density, angles-of-attack and yaw angles of -10-, 0-,

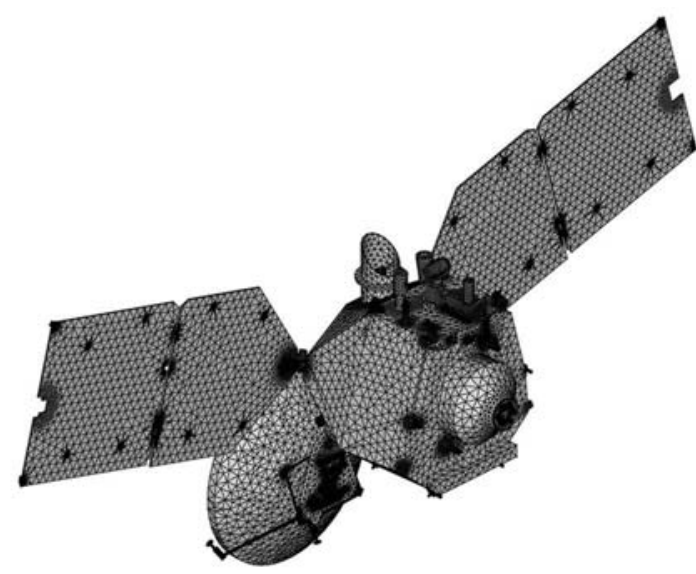

Figure 2. Computational geometry model.

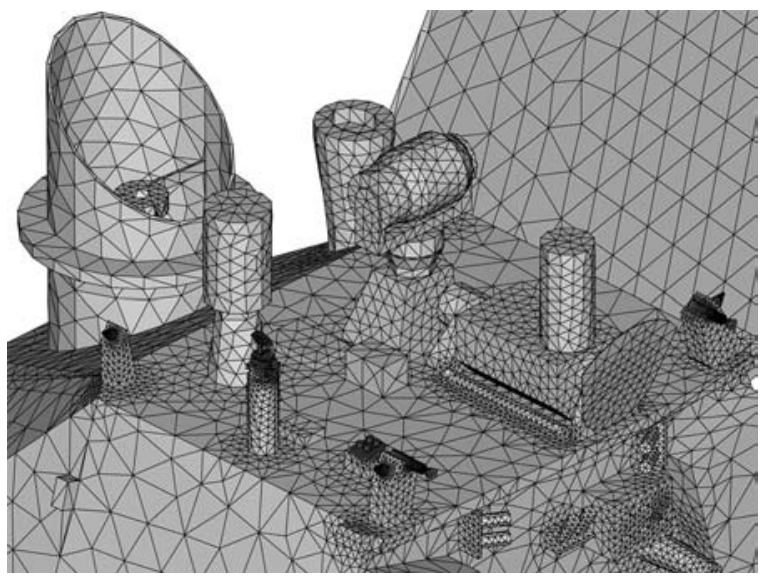

Figure 3. Science deck close-up.

2 of 8

American Institute of Aeronautics and Astronautics Paper 2006-3890 
and $+10-$ deg were also simulated. The estimated uncertainty on the value of $C_{h}$ was approximately $10 \%$ based on parametric sensitivity studies or direct data from various sources of uncertainty arising from computational errors, physical modeling errors, and boundary condition errors.

Free molecular results were obtained using DACfree. ${ }^{4}$ DACfree is a companion code to DAC, which utilizes the same unstructured triangular surface mesh. The free molecular forces, moments, and heat transfer rates are calculated with analytical free-molecular analysis and line-of-sight shadowing.

Table 1. Free stream conditions.

\begin{tabular}{ccccc}
\hline \hline$n_{\infty}$ & $\rho_{\infty}\left(\mathrm{kg} / \mathrm{m}^{3}\right)$ & $V_{\infty}(\mathrm{m} / \mathrm{s})$ & $T_{\infty}(\mathrm{K})$ & $\lambda_{\infty}(\mathrm{m})$ \\
\hline $7.795 \times 10^{16}$ & $5.6007 \times 10^{-9}$ & 4811 & 144.77 & 7.63 \\
$1.392 \times 10^{17}$ & $1.0001 \times 10^{-8}$ & 4811 & 144.77 & 4.27 \\
$2.475 \times 10^{17}$ & $1.7783 \times 10^{-8}$ & 4811 & 144.77 & 2.40 \\
$4.402 \times 10^{17}$ & $3.1628 \times 10^{-8}$ & 4811 & 144.77 & 1.35 \\
$1.392 \times 10^{18}$ & $1.0001 \times 10^{-7}$ & 4811 & 144.77 & 0.42 \\
$2.088 \times 10^{18}$ & $1.5002 \times 10^{-7}$ & 4211 & 144.77 & 0.28 \\
$3.480 \times 10^{18}$ & $2.5004 \times 10^{-7}$ & 3911 & 144.77 & 0.17 \\
$4.872 \times 10^{18}$ & $3.5005 \times 10^{-7}$ & 3611 & 144.77 & 0.12 \\
\hline \hline
\end{tabular}

\section{Results}

\section{A. Effect of Chemical Reactions}

The importance of including chemical reactions must first be analyzed. A 2-specie, non-reacting chemistry model was compared to a 9-specie, reacting chemistry model at the highest expected density to be experienced in flight $\left(32 \mathrm{~kg} / \mathrm{km}^{3}\right)$. The variation of the non-dimensional incident heating coefficient, $C_{h}$, is compared over the windward side of the MRO for the reacting and non-reacting cases in Fig. 4. Data was then extracted from the center-line of a solar panel from the inboard root of the panel to the outboard edge. These data are presented in Fig. 5. The incident heating rates between the reacting and non-reacting flow cases differed by only a couple of percentage points. Therefore, it was determined that the addition of chemical reactions does not significantly effect the heating rate and was not included in the database.

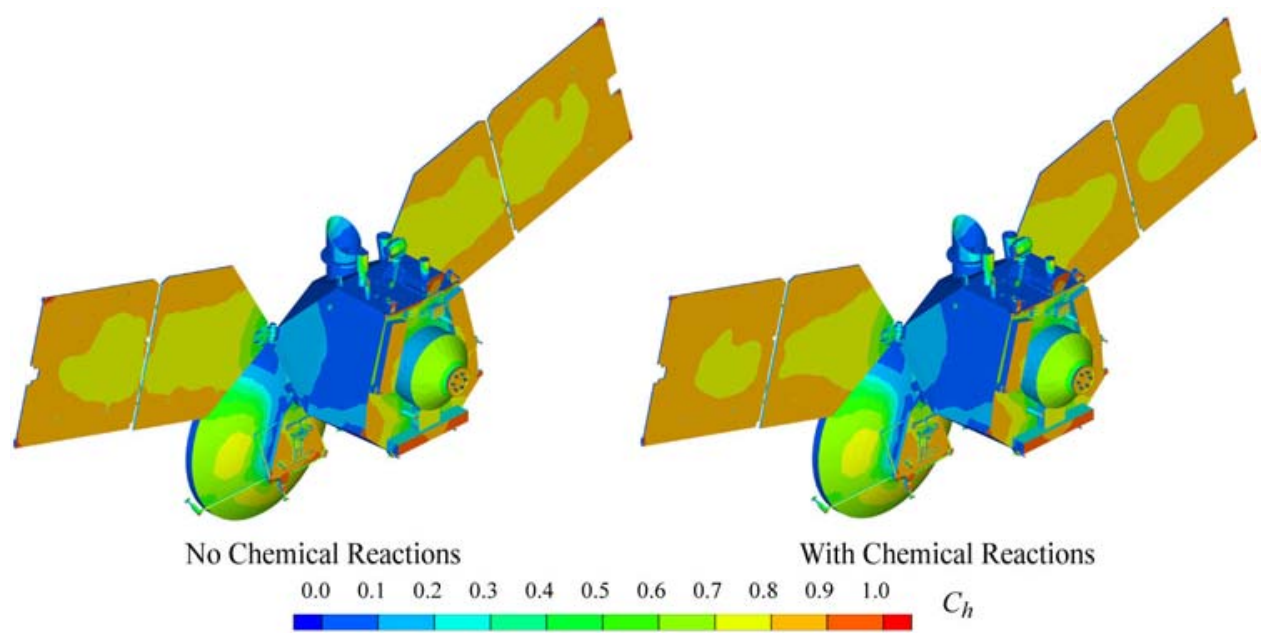

Figure 4. Global effect of chemical reactions on incident heating at $\rho=32 \mathrm{~kg} / \mathrm{km}^{3}, \alpha=0-\mathrm{deg}, \beta=0$-deg. 3 of 8 


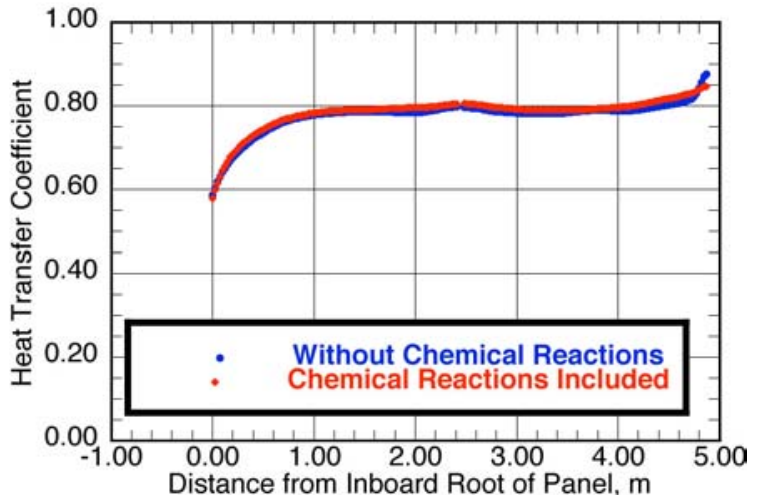

Figure 5. Comparison of incident heating along the solar panel center-line with and without chemistry effects.

\section{B. Effect of Density}

Due to the elliptic orbit of the MRO at the beginning of the aerobraking phase of the mission, a range of densities will be encountered. As a lower limit, free-molecular simulations were examined. The nominal maximum density that the MRO will encounter during aerobraking will be about $32 \mathrm{~kg} / \mathrm{km}^{3}$. Simulations of approximately $6,10,18,32$, $100,150,250$, and $350 \mathrm{~kg} / \mathrm{km}^{3}$, as well as free-molecular, were carried out in order to quantify the effect of the varying density along the trajectory. Heating results for these densities at the nominal angle-of-attack and side-slip angle of 0-deg are presented in Fig. 6. Although the non-dimensionalized heating may be decreasing as density increases, it should be noted that the actual heating rates are increasing since the density is also increasing. The value of $C_{h}$ decreases with density because the kinetic energy of the incident molecules is decreasing due to the increasing number of collisions in the flow.

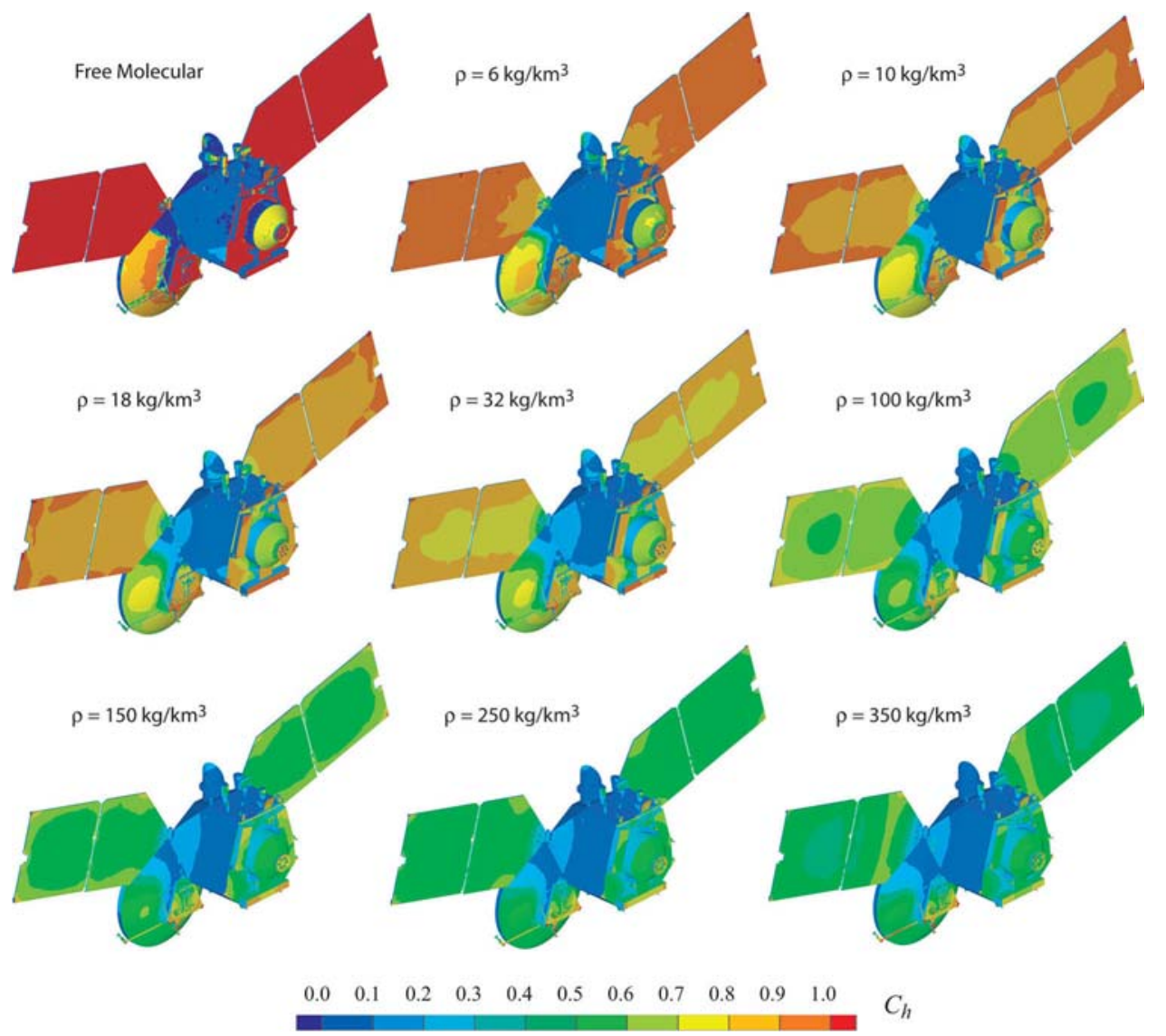

Figure 6. Effect of density at $\alpha=0$-deg, $\beta=0$-deg.

4 of 8

American Institute of Aeronautics and Astronautics Paper 2006-3890 


\section{Effect of Angle-of-Attack and Side-slip}

A variety of angles-of-attack and side-slip angles were examined in this study. Values of -10-, 0-, and +10-deg were simulated for both of these parameters. A sample of these angles are presented in Fig. 7 at the maximum nominal density of $32 \mathrm{~kg} / \mathrm{km}^{3}$. The MRO passes through the atmosphere with the science instruments pointed downward towards the surface of Mars, so in Fig. 7, Mars is "up." A positive angle-of-attack has therefore been defined as having the MRO as seen in Fig. 7 rotate clockwise so that the science instruments are more directly into the flow (the edge of the solar panels that experiences higher heating is pointed more directly into the wind).

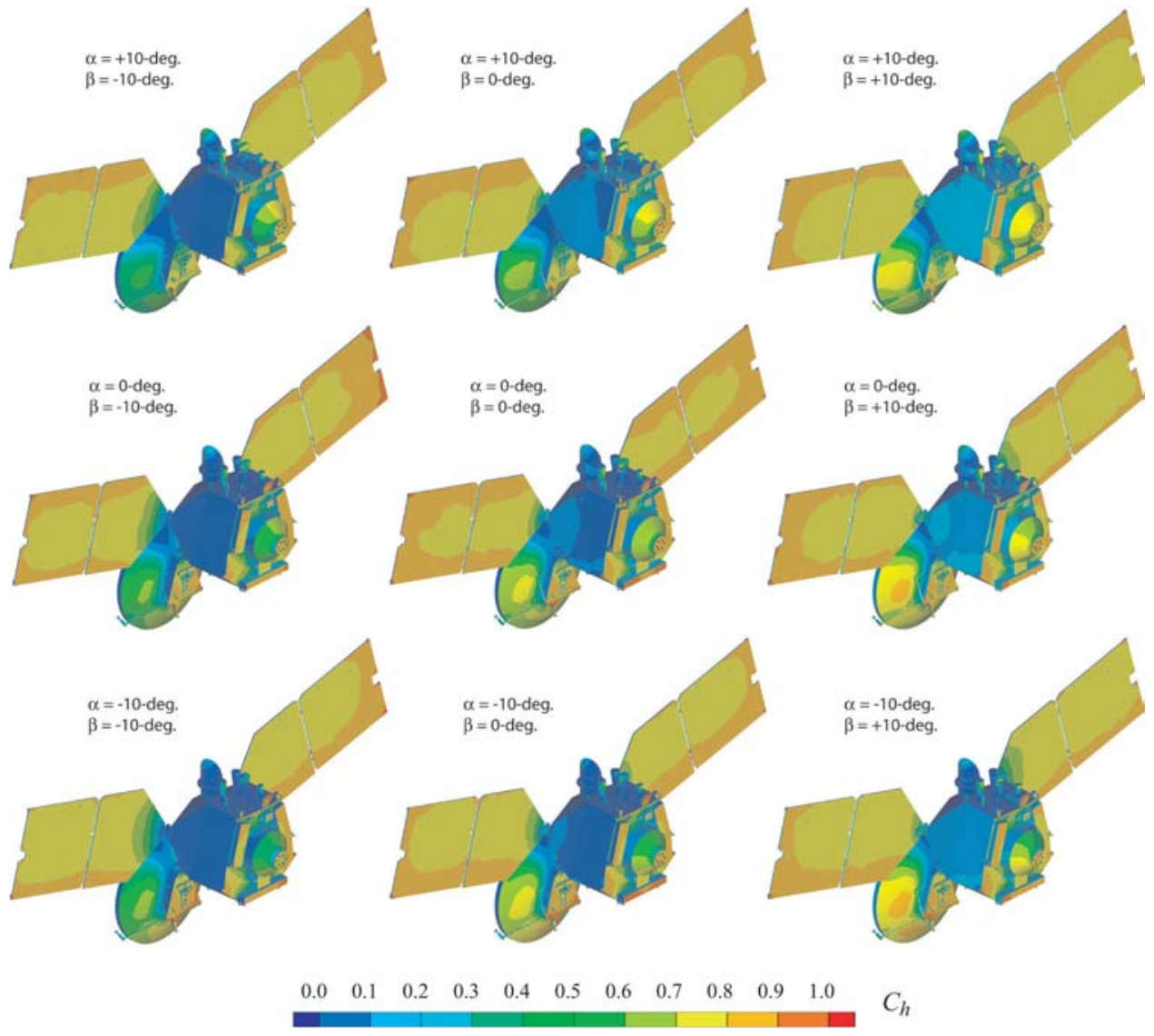

Figure 7. Effect of angle-of-attack and yaw at $\rho_{\infty}=32 \mathrm{~kg} / \mathrm{km}^{3}$.

\section{Comparison to Flight Data}

As the Mars Reconnaissance Orbiter performs the aerobraking maneuver, engineers continually monitor flight data sent back to Earth to make sure that the MRO is going to complete aerobraking on time in the safest possible manner. One aspect of this monitoring process is calculating the surface heat flux from surface temperature sensor data and comparing the calculated in-flight heat flux with the predicted pre-flight heat flux from the aeroheating database. Three sensor locations on one of the solar panels are being monitored. The sensor locations can be seen in Fig. 8. The red outlined sensors (T-0109, T-0110, and T-0309) are the forward-facing temperature sensors. The blue outlined sensor (T-0310) is the only temperature sensor on the back face of the solar array being monitored.

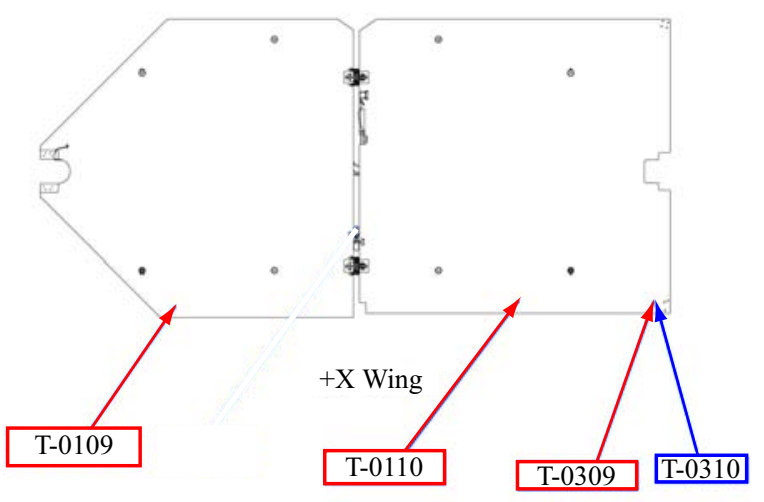

Figure 8. Temperature sensor locations. 
The heat flux due to atmospheric heating to the solar arrays at the three forward-facing temperature sensor locations was calculated by modifying the code $1 \mathrm{DHEAT}^{5}$, which is a one-dimensional heat transfer analysis tool. The finite-volume implementation of 1DHEAT takes direct account of the variable substrate thermal properties and removes the restriction of a semi-infinite substrate (thus allowing for a multiple layer analysis), which is important due to the structure of the solar panels. The code was modified to allow for prescribing the temperature on the back surface of the solar panels as a function of time and to allow for radiative boundary conditions on the front and back sides of the solar panels, including the orbital flux (solar, albedo, and Mars IR) input and radiative emission of energy for given absorptivity and emissivity, respectively.

The material and the corresponding thickness for each layer of the solar array can be seen in Table 2. There are a total of five layers to be accounted for (adhesives are neglected in this analysis). It should be noted that the total thickness of the inboard panel is greater than that of the outboard panel. The solar cell material thermal properties are presented in Table 3.

Table 2. Material and thickness of solar array layers.

\begin{tabular}{cccc}
\hline \hline Layer & Material & $\begin{array}{c}\text { Thickness }(\mathrm{m}) \\
(\mathrm{T}-0190)\end{array}$ & $\begin{array}{c}\text { Thickness }(\mathrm{m}) \\
(\mathrm{T}-0110, \mathrm{~T}-0309)\end{array}$ \\
\hline 1 & Kapton & $7.6 \times 10^{-5}$ & $7.6 \times 10^{-5}$ \\
2 & M55 J/RS-3 & $5.0 \times 10^{-4}$ & $2.5 \times 10^{-4}$ \\
3 & Al HC 1.6 PCF & $2.9 \times 10^{-2}$ & $2.26 \times 10^{-2}$ \\
4 & M55J/RS-3 & $5.0 \times 10^{-4}$ & $2.5 \times 10^{-4}$ \\
5 & Kapton & $5.0 \times 10^{-5}$ & $5.0 \times 10^{-5}$ \\
\hline \hline
\end{tabular}

Table 3. Solar cell material properties.

\begin{tabular}{ccccc}
\hline \hline Material & $\rho_{\mathrm{s}}\left(\mathrm{kg} / \mathrm{m}^{3}\right)$ & $c_{p}(\mathrm{~J} / \mathrm{kg} \mathrm{K})$ & $k(\mathrm{~W} / \mathrm{m} \mathrm{K})$ & $\varepsilon$ \\
\hline Kapton & 1450 & 1000 & 0.1557 & 0.75 \\
M55 J/RS-3 & 1700 & 795 & 1.0 & -- \\
$\mathrm{Al} \mathrm{HC} 1.6 \mathrm{PCF}$ & 28 & 830 & 0.9 & -- \\
\hline \hline
\end{tabular}

The measured surface temperatures of the three forward-facing temperature sensors as well as the estimated radiative heat flux to each sensor are presented in Fig. 9. The radiative heat flux was estimated by the Langley MRO Thermal Team by taking into account inputs from the Sun, Mars, and solar albedo as the spacecraft orbits Mars. There was only one temperature sensor available for the rear-facing boundary conditions, T-0310, which is located almost directly on the other side of the solar panel from T-0309. In order to obtain reasonable back-face temperatures

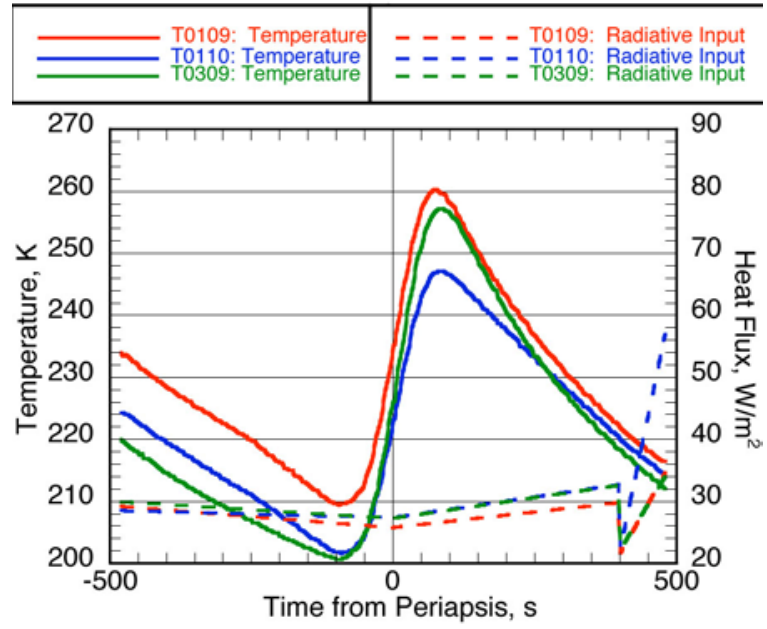

Figure 9. Surface temperature and radiative heat flux of front-facing temperature sensors (orbit 27).

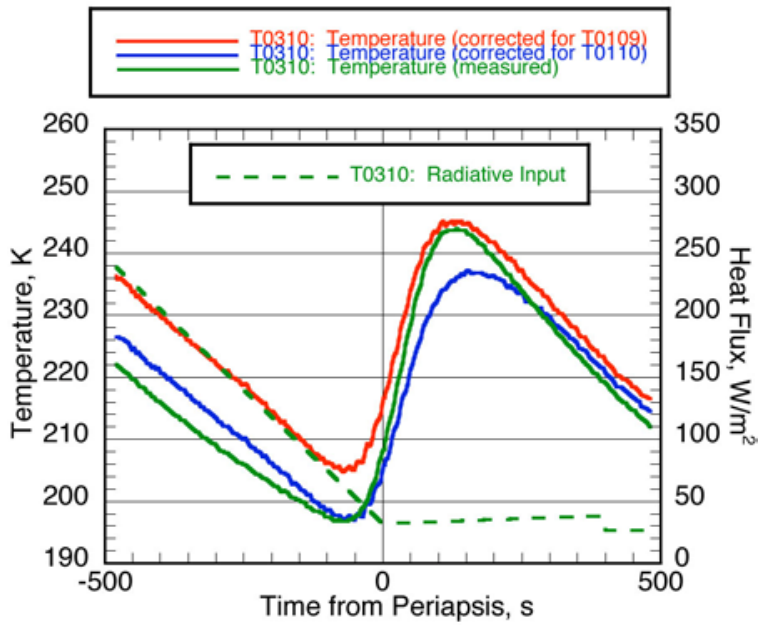

Figure 10. Surface temperature and radiative heat flux of back-facing temperature sensors (orbit 27).

6 of 8

American Institute of Aeronautics and Astronautics Paper 2006-3890 


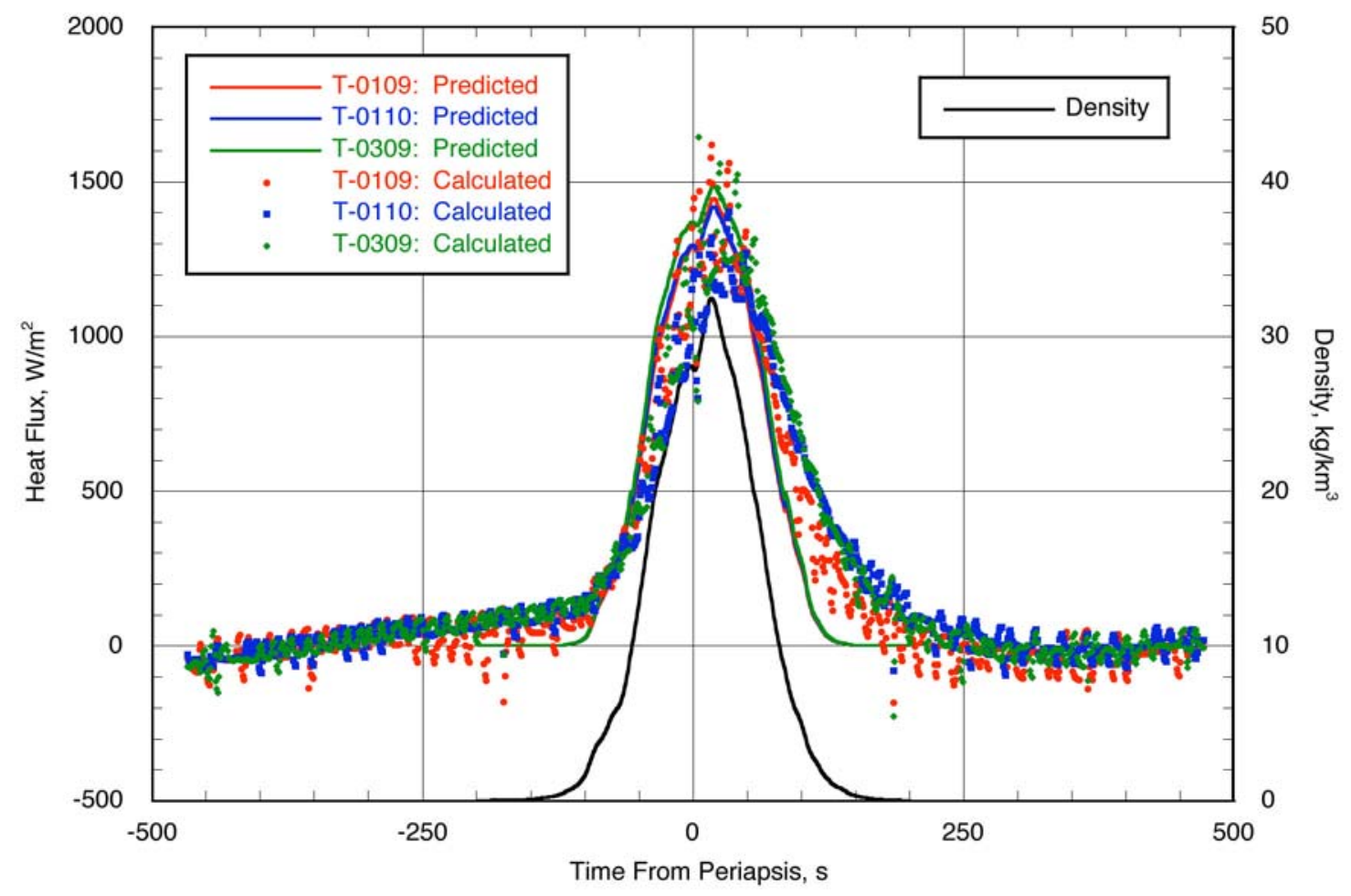

Figure 11. Comparison of predicted and computed convective heat flux (orbit 27).

for T-0109 and T-0110, it was assumed that the back-surface temperature distributions roughly resembled the forward-surface temperature distributions, anchored to T-0310. Therefore, for example, to calculate the back-face temperature for T- 0109 , the temperature at T-0310 was multiplied by the ratio of the temperatures at T-0109 and T-0309. The radiative heat flux at T-0310 was used for the back-face radiative inputs for T-0109 and T-0110, as well. The back-face temperatures and heat flux used are presented in Fig. 10.

The calculated in-flight heat flux at the three front facing temperature sensors, along with the predicted pre-flight values from the database and density, are presented in Fig. 11. The predicted heat flux was interpolated from the database as a function of spacecraft orientation and density by way of a second order Lagrangian interpolation subroutine. Outside of the atmosphere (for this orbit, the spacecraft is within the sensible atmosphere between approximately 150 seconds before and after periapsis), the convective heating should be equal to zero. The values that were calculated for orbit 27 were within $\pm 100 \mathrm{~W} / \mathrm{m}^{2}$ of the expected value of zero with a positive slope to the heat flux as the MRO approached the atmosphere. This may be due to slightly incorrect predicted values of orbital heat flux or some variability in material thermal properties (incorrect values or values that change with temperature). Part of this discrepancy could also be due to the change in temperature that the solar panels are radiating to. The value pre-set in this study was $10 \mathrm{~K}$, but if this value were increasing with time, the heating rate would decrease along the trajectory. Another cosideration is that the code used in this analysis uses the assumptoin of one-dimensional conduction. If there are any temperature gradients span-wise across the solar panels, the accuracy of the computed heat flux will decrease.

The computed heat flux also appears to lag behind the predicted values of heat flux as the spacecraft passes through the atmosphere, which may also be attributable to slightly incorrect thermal properties or thermal gradients. The calculated peak heating rate was about $15-20 \%$ lower than the predicted value. This could either once again be due to thermal properties, thermal gradients, or due to the fact that the accommodation coefficient for a spacecraft is almost always less than one, which was used in the development of the database. The lower the accommodation coefficient, the lower the convective heating will be. However, due to the uncertainties in the boundary conditions and structural thermal properties, one must be careful in drawing conclusions such as these based on such a small difference in heating rates and the amount of noise in the data.

\section{7 of 8}

American Institute of Aeronautics and Astronautics Paper 2006-3890 


\section{Conclusion}

An aeroheating database has been developed for the aerobraking phase of the Mars Reconnaissance Orbiter's mission. Effects of chemistry, density, and spacecraft orientation were examined. It was determined that the addition of chemical reactions did not significantly change the heating rate of the spacecraft, therefore was not included in the development of the database. A total of 81 simulations were performed to define the spacecraft aeroheating in terms of variations in density, angle-of-attack, and side-slip angle.

Computational results have been compared to flight data. Several sources of uncertainty have been identified that could have potentially contributed to the discrepancies seen between predicted aeroheating values and those calculated from flight temperature sensor data. These uncertainties include values of thermal properties, possible thermal gradients span-wise across the solar panels, radiative input values, and the temperature to which the solar panel radiates to. Although there are discrepancies between the predicted and calculated heat rates, the overall agreement is favorable. The calculated peak heating rate was about $15-20 \%$ lower than the predicted value. Given the predicted uncertainties for the aeroheating database along with the uncertainties associated with the calculation of the heating rates, the predicted and calculated heating rates agree favorably.

\section{References}

${ }^{1}$ Wilmoth, R.G., LeBeau, G. J., and Carlson, A. B., "DSMC Grid Methodologies for Computing Low-Density, Hypersonic Flows About Reusable Launch Vehicle,” AIAA Paper 96-1812, June 1996.

${ }^{2}$ LeBeau, G. J. and Lumpkin III, F. E., "Application Highlights of the DSMC Analysis Code (DAC) Software for Simulating Rarefied Flows," Computer Methods in Applied Mechanics and Engineering, Vol. 191, Issues 6-7, December 7, 2001, Pages 595609.

${ }^{3}$ Bird, G. A., Molecular Gas Dynamics and the Direct Simulation of Gas Flows, Clarendon Press, Oxford, 1994.

${ }^{4}$ Moss, J. N., Blanchard, R. C., Wilmoth, R. G. and Braun, R. D., "Mars Pathfinder Rarefied Aerodynamics: Computation and Measurements," Journal of Spacecraft and Rockets, Vol. 36, No. 3, May-June 1999, pp. 399-404.

${ }^{5}$ Hollis, B.R., "User's Manual for the One-Dimensional Hypersonic Experimental Aero-Thermodynamic (1DHEAT) Data Reduction Code," NASA CR-4691, Aug. 1995. 